\title{
A parts-based approach for automatic 3D-shape categorization using belief functions
}

Hedi Tabia, University Lille 1

Mohamed Daoudi, TELECOM Lille 1

Jean-Philippe Vandeborre, TELECOM Lille 1

Olivier Colot, University Lille 1

Grouping 3D-objects into (semantically) meaningful categories is a challenging and important problem in $3 \mathrm{D}$-mining and shape processing. Here, we present a novel approach to categorize 3D-objects. The method described in this paper, is a belief function based approach and consists of two stages. The training stage, where 3D-objects in the same category are processed and a set of representative parts is constructed, and the labeling stage, where unknown objects are categorized. The experimental results obtained on the ToscaSumner and the Shrec07 datasets show that the system efficiently performs in categorizing 3D-models.

Categories and Subject Descriptors: H.3.1 [Information Storage and Retrieval]: Content Analysis and Indexing; I.2.10 [Artificial Intelligence]: Vision and Scene Understanding-shape; I.5.3 [Pattern Recognition]: Clustering

General Terms: Multimedia data, Classification

Additional Key Words and Phrases: 3D-categorization, object recognition, belief functions

\section{INTRODUCTION}

The representation of objects in three dimensions (3D) has recently have recently gained great popularity in computer vision communities and has become an integral part of modern computer graphics applications, such as computer-aided design, game development and more recently film production. At the same time, 3D data have become widely used in diverse fields including computer vision, computational geometry, molecular biology and medicine. The availability of low cost 3D scanners and the rapid evolution in graphics hardware and software, has greatly facilitated 3D model acquisition, creation and manipulation. The content-based analysis becomes a necessary solution for structuring, managing and organizing 3D objects from large collections. In this context, the aim of this paper is to propose a fully automatic framework for $3 \mathrm{D}$-object categorization based on the content.

\subsection{Related works}

Through the state-of-the-art, we can find mainly two different families of related work. The first family deals with the 3D-shape retrieval and the second family is concerned by the $3 \mathrm{D}$-shape classification.

H. Tabia and O. Colot are with LAGIS UMR CNRS 8146 / University Lille 1, Cite Scientifique 59655 Villeneuve d'Ascq Cedex, France

M. Daoudi and J-P. Vandeborre are with TELECOM Lille 1; Institut TELECOM / LIFL UMR CNRS 8022 / University Lille 1, Cite Scientifique 59655 Villeneuve d'Ascq Cedex, France.

Permission to make digital or hard copies of part or all of this work for personal or classroom use is granted without fee provided that copies are not made or distributed for profit or commercial advantage and that copies show this notice on the first page or initial screen of a display along with the full citation. Copyrights for components of this work owned by others than ACM must be honored. Abstracting with credit is permitted. To copy otherwise, to republish, to post on servers, to redistribute to lists, or to use any component of this work in other works requires prior specific permission and/or a fee. Permissions may be requested from Publications Dept., ACM, Inc., 2 Penn Plaza, Suite 701, New York, NY 10121-0701 USA, fax +1 (212) 869-0481, or permissions@acm.org.

(c) YYYY ACM 1539-9087/YYYY/01-ARTA $\$ 10.00$

DOI 10.1145/0000000.0000000 http://doi.acm.org/10.1145/0000000.0000000 
In the first family, many systems have been proposed for efficient information retrieval from digital collection of 3D-objects. Kazhdan et al. [Kazhdan et al. 2003] present a general approach based on spherical harmonics for obtaining rotation invariant representations and show its application in the retrieval task. Antini et al. [Antini et al. 2005] present an approach relying on curvature correlograms to perform description and retrieval by content of 3D objects. Funkhouser et al. [Funkhouser et al. 2003] present a web-based search engine system that supports queries based on 3D sketches, 2D sketches, 3D models, and/or text keywords. For the shape-based queries, they have developed a matching algorithm that uses spherical harmonics to compute discriminating similarity measures without requiring repair of model degeneracies or alignment of orientations. Filali et al. [Filali Ansary et al. 2007] propose a method for $3 \mathrm{D}$-object indexing based on $2 \mathrm{D}$-views. In their paper, they present an adaptive nearest neighbor-like algorithm to select the most relevant characteristic views from the 3D-object. A Bayesian approach is used in order to improve the retrieval rate. Assfalg et al. [Assfalg et al. 2007] presents an adaptive spin images approach for 3D-retrieval by content. Hilaga et al. [Hilaga et al. 2001] proposed a technique, called Topology Matching, in which similarity between polyhedral models is calculated by comparing Multiresolutional Reeb Graphs (MRGs) based on the Reeb graph computation of a 3Dobject. Bronstein et al. [Bronstein et al. 2008; Bronstein et al. 2009] also proposed to consider the 3D-matching problem as a multi-criterion optimization problem trying to simultaneously maximize the similarity and the significance of the matching parts.

Compared to the work on 3D-object retrieval where objects are compared together in pair, 3D-classification, which consists to affect an object query to one category, is still an open problem. Few paper such as [Huber et al. 2004; Donamukkala et al. 2005] have addressed this issue. The method is used for classifying vehicles into a set of pre-determined object classes. For that, parts are extracted from training objects and grouped into part classes. A mapping from part classes to object classes is derived from the learned part classes and known object classes. For a 3D object, after local shape features are computed, the object class is determined using the learned part classes and the part-to-object mapping. The decomposition in the approach is just to divide objects into front, middle, and back parts. The method is based on a Bayesian classifier and it is limited to specific datasets, which should respect the decomposition constraint.

\subsection{Main contributions}

In this paper, we present a fully automatic framework for 3D-object categorizing based on the belief functions. The categorization is addressed with a parts-based approach. It consists of capturing a compact model of a given category by building a set of representative parts. For this purpose, the objects in the same category are partitioned into several parts. These parts are then used to construct a set of representative ones with which objects in the same category can be described. A straightforward way to build this set is using vector quantization techniques. Here, we use a variation of the evidential k-nearest neighbors algorithm. The centroids of the resulting clusters are used as representative parts of the category. This process is iterated for all the categories in the training set.

The labeling of unknown 3D-objects is achieved by labeling their associated parts. Here, we assume that each part can help to predict the category of the whole object. More specifically, each part of the object to be labeled is considered as an item of evidence supporting certain hypotheses concerning the category membership of that object. Based on this evidence, the object parts are compared with category representative parts and basic belief masses are assigned to each category. As a result of considering each object part in turn, we obtain a set of Basic Belief Assignments (BBAs) that 


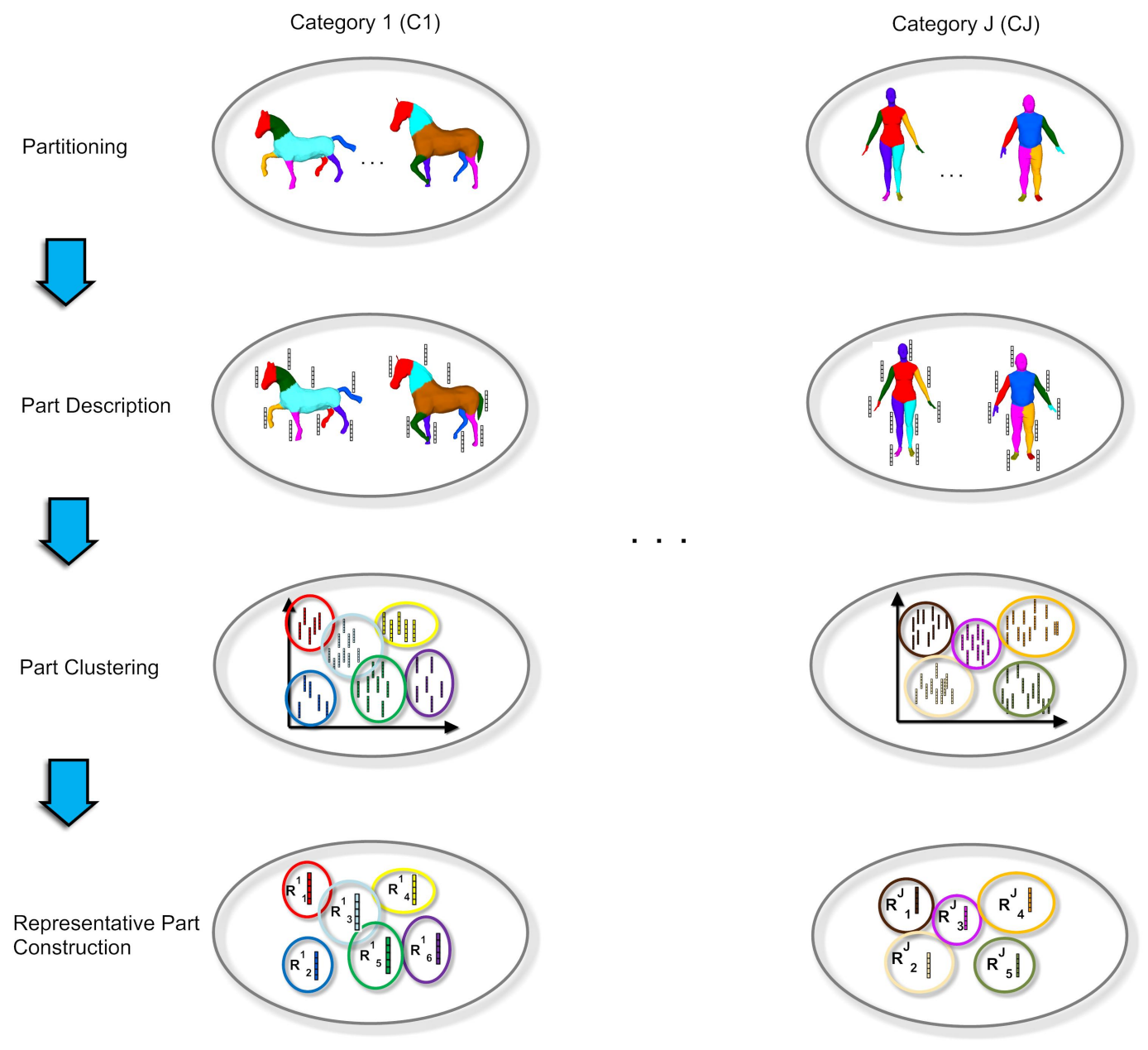

Fig. 1. The training system architecture: the input of the system is categories of 3D-objects. The output is a set of representative-parts. The objects in this figure are selected from the animal and the human categories in the Shrec07 dataset.

can be combined using the Dempster's rule of combination to form a resulting BBA synthesizing a final belief regarding the category of the whole object.

Another issue is dealt with in this paper, is when labeling an unknown 3D-object, one can be faced with the problem of handling an unclassifiable object (reject). Here we show that we are able to handle this issue using belief theory based data association method. Using a specific modeling of belief functions, this is done by detecting and managing a portion of a conflict, which originates from the non-exhaustivity of the frame of discernment.

The remainder of the paper is organized as follows. In Section 2 the training stage is presented. Then, in Section 3 the labeling stage is detailed. Section 4 presents experimental results. Conclusions and future developments end the paper. 


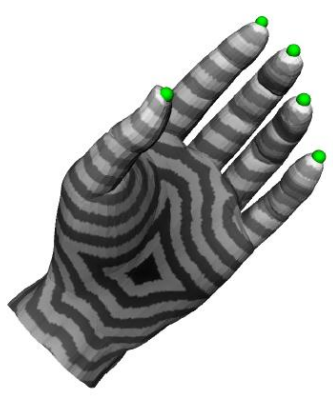

(a)

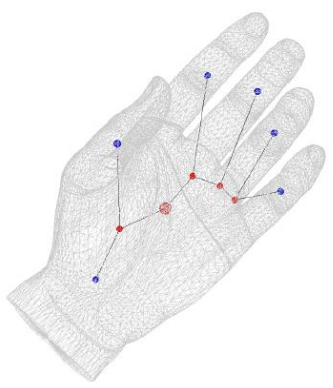

(b)

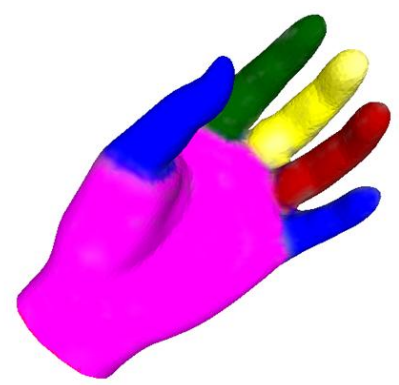

(c)

Fig. 2. 3D-object partitioning using topology driven 3D mesh segmentation algorithm

\section{A 3D-OBJECT CATEGORY MODEL}

From a training set of 3D-objects, the procedure we suggest for obtaining the model of each category is based on the assumption that 3D-objects in the same category have the same parts. More specifically, we assume that each category can be represented by a set of representative parts, with which shapes in that category can be described. In this section, we focus on constructing the set of representative parts for each category. Figure 1 presents the different steps of the training process. Given a set of $J$ categories, The Figure shows four main steps applied for each category: (1) object partitioning; (2) part description; (3) part clustering; (4) representative part computation.

\subsection{Object partitioning and part description}

3D-partitioning, which consists to extract a set of parts that share the same characteristics from a 3D-object, is a deeply-studied domain as the reader may realize through a recent comprehensive survey [Shamir 2008]. In our method, we adopt a fully automatic topology driven 3D mesh segmentation algorithm [Tierny et al. 2007] where feature boundaries and feature hierarchy are both computed in a semantic-oriented manner. The main steps of this approach are shown in Figure 2. First, a set of feature points is extracted from the 3D object as shown in Figure 2(a). Then the enhanced topological skeleton of the input triangulated surface is constructed (Figure 2(b)). Finally, it is used to delimit the core of the object and to identify junction areas. This final step results in a fine segmentation of the object (Figure 2(c)).

After the object partitioning, parts are represented by local descriptors which capture the geometry of the part. It is well known that the performance of all data classifier depends critically on the ability of the descriptors to discriminate among the various classes. Choosing the right set of feature is a difficult problem. The descriptors must be sufficiently rich to discriminate between different parts of shapes, and at the same time be invariant to different transformations that a shape can undergo. There exists several part description algorithms, and below we overview some of them.

- GD2: The GD2 is a local distributions of geodesic distances. It has been used for shape recognition by Osada et al. [Osada et al. 2002] as a global shape distribution, which measures Euclidean distances of random surface points.

- Gcords: Geodesic cords-based descriptors is defined as a distribution of geodesic distances from one source point to other. First, an Euclidean Cords-based descriptors was be introduced by Paquet et al. [Paquet and Rioux 1999] for global 3D-shape matching. We have adapted this approach to local feature descriptor. 
- HSI: Histogram of Shape Index, is defined as the histogram of shape index values, calculated over mesh part. The shape index, first introduced by Koenderinket al. [Koenderink and van Doorn 1992], is defined as a function of the two principal curvatures on continuous surfaces.

\subsection{Representative part construction}

Here, we attempt to find representative parts of each category. For this end, we developed an evidential clustering method based on the Transferable Belief Model (TBM) concept [Shafer 1976; Zouhal and Denoeux 1998].

2.2.1. Transferable Belief Model concept. The TBM is based on a two-level model: a credal level where beliefs are entertained, combined and updated, and a pignistic level where beliefs are converted into probabilities to make decisions.

- Credal level: Let $\Omega$ denote a finite set called the frame of discernment. A Basic Belief Assignment (BBA) or mass function is a function $m: 2^{\Omega} \rightarrow[0,1]$, such that: $\sum_{A \subseteq \Omega} m(A)=1 . m(A)$ measures the amount of belief that is committed to $\mathrm{A}$. The subsets $\mathrm{A}$ of $\Omega$ such that $m(A)>0$ are called focal elements.

Given two BBAs $m_{1}$ and $m_{2}$ defined over the same frame of discernment $\Omega$ and induced by two distinct pieces of information, we can combine them using the Dempster's combination rule [Shafer 1976] given by:

$$
\left(m_{1} \otimes m_{2}\right)(A)=\frac{1}{1-\sum_{B \cap C=\emptyset} m_{1}(B) m_{2}(C)} \sum_{B \cap C=A} m_{1}(B) m_{2}(C) .
$$

for all $A \subseteq \Omega$.

- Pignistic level: When a decision has to be made, the beliefs held at the credal level induce a probability measure at the pignistic level. Hence, a transformation from belief functions to probability functions must be done. This transformation is called the pignistic transformation. Let $m$ be a BBA defined on $\Omega$, the probability function induced by $m$ at the pignistic level, denoted by $\operatorname{Bet} P$ and also defined on $\Omega$ is given by:

$$
\operatorname{BetP}(\omega)=\sum_{A \subseteq \Omega, \omega \in A} \frac{m(A)}{|A|}
$$

for all $\omega \in \Omega$ and where $|A|$ is the number of elements of $\Omega$ in $\mathrm{A}$.

2.2.2. Clustering algorithm. Let us consider a category $C$ in the training set. All objects in $C$ are processed (each object is partitioned) and the sets of parts are extracted. Let $P^{C}=\left\{P_{1}, \ldots, P_{N}\right\}$ be the collection of all object parts in $C$. We assume that these parts can be classified into $M$ classes $W=\left\{W_{1}, \ldots, W_{M}\right\}$ (for the choice of $M$ see Algorithm 1)and each part $P_{i}$ will be assumed to possess a class label indicating with certainty its membership to one class in $W$. Let $P_{s}$ be an incoming part to be classified. Classifying $P_{s}$ means assigning it to one class in $W$. Using the vocabulary of the evidential theory, $W$ can be called the frame of discernment of the problem.

Let us denote by $\Theta_{s}$ the set of the k-nearest neighbors of $P_{s}$ in $P^{C}$, according to some distance measure $D$ (in this paper $D$ represents the $L_{2}$ distance between 2 descriptors). Let $P_{k} \in \Theta_{s}$ a 3D-part classed in $W_{l}$. The pair $\left(P_{k}, W_{l}\right)$ can be regarded as a piece of evidence that increases our belief that $P_{s}$ also belongs to $W_{l}$. However, this piece of evidence does not by itself provide $100 \%$ certainty. In the evidential formalism, this can be expressed by saying that only some parts of our belief are committed to $W_{l}$. Since the fact that $P_{k} \in W_{l}$ does not point to any other particular hypothesis, the rest of our belief can not be distributed to anything else than $W$, the whole frame 
of discernment. This item of evidence can therefore be represented by a Basic Belief Assignment (BBA) $m_{k}$ verifying:

$$
\begin{gathered}
m_{k}\left(W_{l}\right)=\chi e^{\left(-\gamma_{l} D^{2}\left(P_{s}, P_{k}\right)\right)} \\
m_{k}(W)=1-m_{k}\left(W_{l}\right) \\
m_{k}(A)=0 \forall A \in 2^{W} \backslash\left\{W,\left\{W_{l}\right\}\right\}
\end{gathered}
$$

With $\chi$ a parameter such that $0 \leq \chi \leq 1 . \gamma_{l}$ is obtained by an optimization procedure proposed by Zouhal and Denoeux [Zouhal and Denoeux 1998]. We set $\chi=0.9$. For each of the k-nearest neighbors of $P_{s}$, a BBA depending on both its class label and its distance to $P_{s}$ can therefore be defined. In order to make a decision regarding the class assignment of $P_{s}$, these BBAs can be combined using Dempster's rule [Shafer 1976] into one BBA $m_{s}$. As a result, $P_{s}$ will take the label of the class maximizing the pignistic probability induced by $m_{s}$. Algorithm 1 summarizes this method.

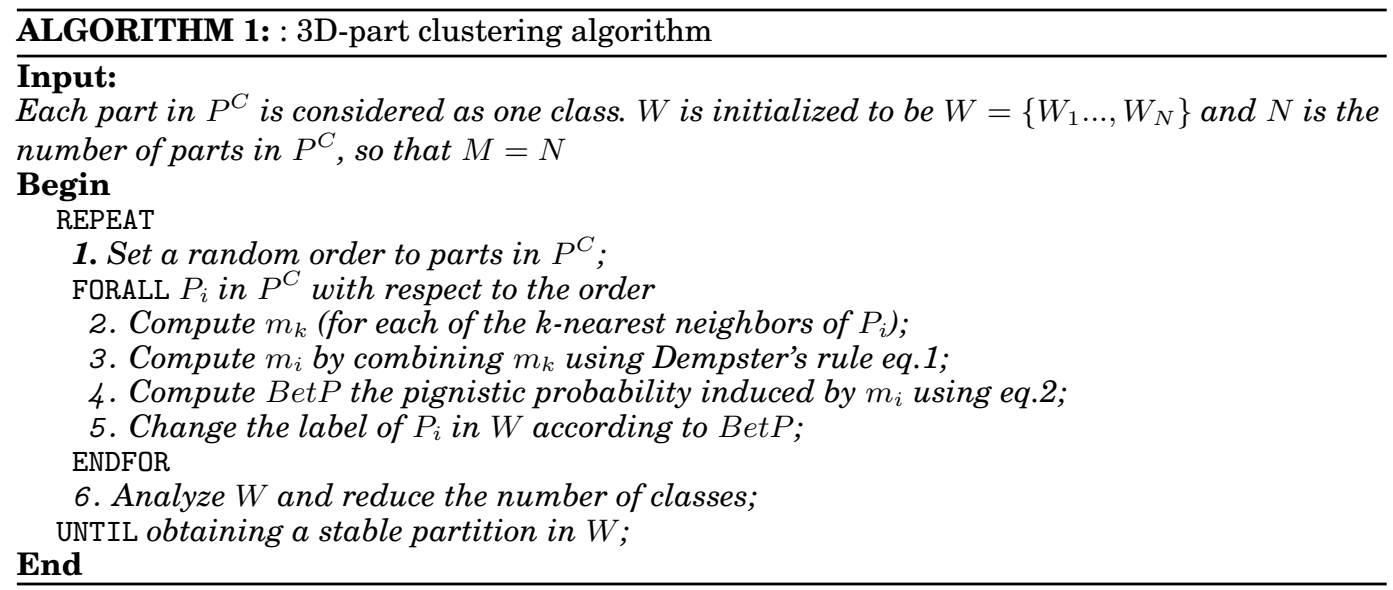

Once the clustering process is achieved, we compute the centroid of each cluster. The centroid is a part whose parameter values are the mean of the parameter values of all the parts in the cluster. Centroids, in this paper, are called representative parts and denoted by $R$.

\section{LABELING A NEW 3D-OBJECT}

In this section, we focus on the labeling of 3D objects. Figure 3 shows the various steps of this process. First, giving an object $O$ to be labeled (Figure 3 step a), the algorithm begins by partitioning this object (Figure 3 step b). Second, an invariant descriptor is associated to each extracted part of that object (Figure 3 step c). The partitioning and the description of these parts are done in the same way as in the training process. The labeling of the object $O$ is achieved based on its parts. Here, we assume that each part can help to predict the category of the whole object. In the context of belief functions, we can say that each part represents an evidence source which provides an information regarding the category of the object. By considering all parts, we obtain a set of evidence sources that can be combined to produce a final decision concerning the category of the object. 


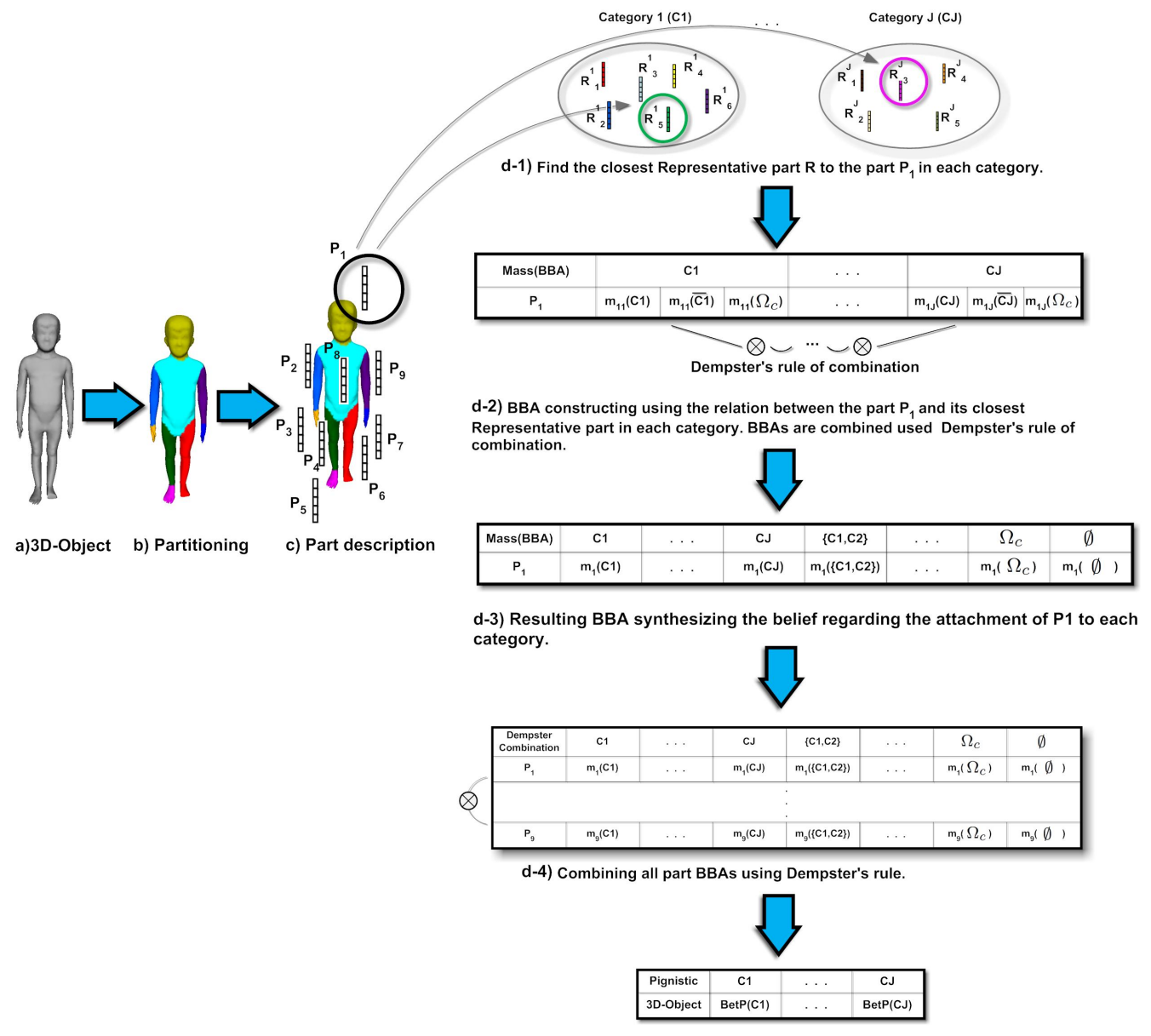

d-5) Labeling the 3D-object using the pignistic probability deduced from the combined BBAs.

Fig. 3. The labeling system architecture: the input of the system is a 3D-object to be labeled. The output is the probabilities of belonging to each category .

\subsection{Evidence extracting from parts}

Recalling here that, after the training process, each category contains a set of representative parts. Given a part extracted from the object to be labeled (for example $P_{1}$ in Figure 3 step c), we select in each category the representative part that is the closest to $P_{1}$ (Figure 3 step d-1). Then, from the selected representative part a mass function that quantifies the degree of belief given to the assumption " $P_{1}$ matches with a particular category $C_{j}$ " is derived (Figure 3 step d-2). As a result of considering each category in turn we obtain a set of BBAs that can be combined using the Dempster's rule of combination to form a resulting BBA. This BBA syntheses a final belief regarding the relation between the part and the categories (Figure 3 step d-3).

More formally, let us denote by $\left\{P_{i}\right\}_{1 \leq i \leq I}$ the set of $I$ parts composing the object $O$. From the training set, we enumerate $J$ categories $\Omega_{c}=\left\{C_{j}\right\}_{1 \leq j \leq J}$. A category $C_{j}$ contains a set of representative parts. Let $R_{P_{i}}^{j}$ be the closest representative part to 
the part $P_{i}$ in the category $C_{j}$. Each pair $\left(P_{i}, R_{P_{i}}^{j}\right)$ with $1 \leq j \leq J$ constitutes a distinct item of evidence regarding the category membership of $P_{i}$. If $P_{i}$ is closed to $R_{P_{i}}^{j}$ then one will be inclined to believe that both parts belong to the same category. On the contrary if their dissimilarity is very large then we consider that $P_{i}$ may belongs to $\overline{C_{j}}$ the complement of the $C_{j}$ in $\Omega_{c}$. Consequently this item of evidence may be postulated to induce a basic belief assignment BBA $m_{i j}$ over $\Omega_{c}$ defined by:

$$
\begin{gathered}
m_{i j}\left(C_{j}\right)=\mu \cdot S\left(P_{i}, C_{j}\right) \\
m_{i j}\left(\overline{C_{j}}\right)=\mu \cdot\left(1-S\left(P_{i}, C_{j}\right)\right) \\
m_{i j}\left(\Omega_{c}\right)=1-\mu
\end{gathered}
$$

$S\left(P_{i}, C_{j}\right)=e^{\left(-D\left(P_{i}, R_{P_{i}}^{j}\right)\right)}$ is a function of the distance between the part $P_{i}$ and its closest representative part $R_{P_{i}}^{j}$ in the category $C_{j} . \mu$ is a weakening coefficient ([Shafer 1976]) associated with the category $C_{j}$. In practice, we set $\mu=0.9$ for all categories.

As a result of considering each category we obtain $J$ BBAs as shown in Figure 3 step d-2. These masses are combined using the Dempster's rule of combination to form a resulting BBA $m_{i}$ synthesizing a final belief regarding the attachment of $P_{i}$ to each category. Figure 3 step d-3 shows the resulting BBA $m_{i}$.

\subsection{D-object labeling}

In order to get a final decision about the category of the unknown 3D-object, all masses $m_{i} i \in[1 . . I]$ are combined using the Dempster's rule of combination (Figure 3 step d-4).

A decision can be made regarding the category membership of the 3D-object by examining the pignistic probability deduced from the resulting mass $m$ (Figure 3 step d-5). The labeling process is summarized in Algorithm 2.

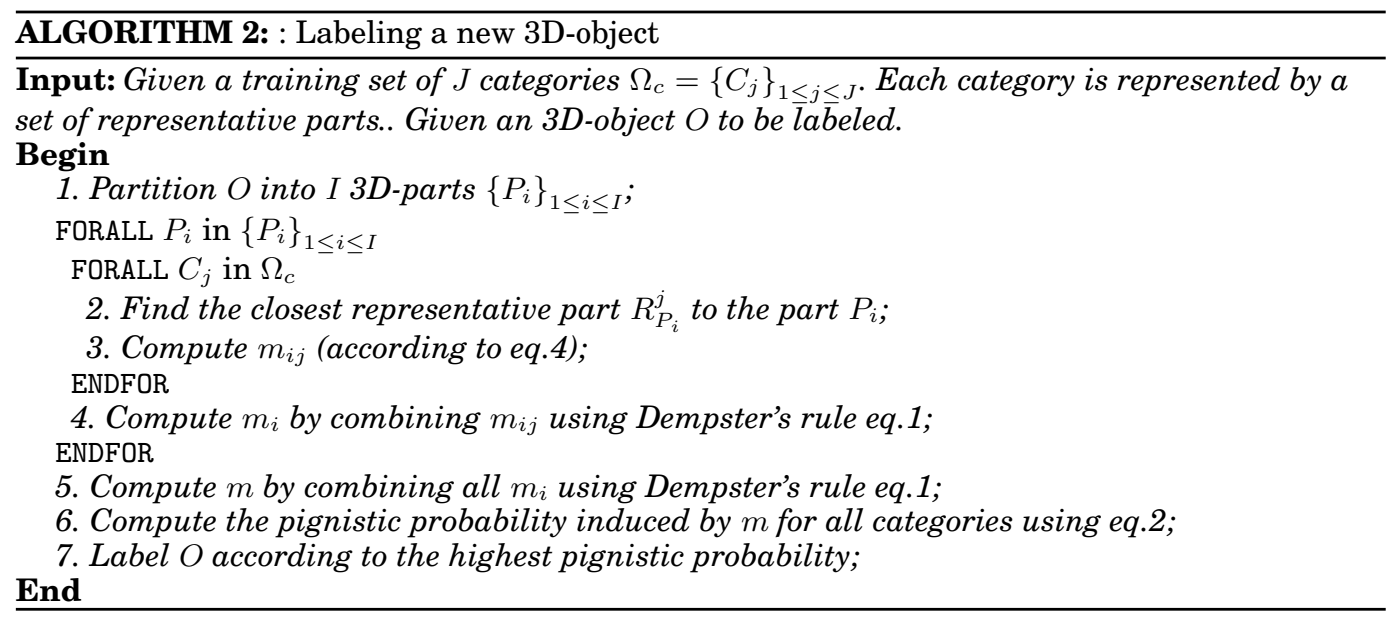

\subsection{Reject option}

Introducing a reject option is very useful, yet a difficult problem in data classification. Instead of Bayesian classifiers where the reject is modeled empirically by comparing the posteriori probability with a threshold (T) [Vailaya et al. 2001], the reject in the belief theory is modeled in natural way. It can be deducted from the conflict on each 


Table I. Representation of
the Dempster's rule of
combination
\begin{tabular}{|c|c|c|c|}
\hline$C_{1}$ & $\emptyset$ & $C_{1}$ & $C_{1}$ \\
\hline$\overline{C_{1}}$ & $C_{2}$ & $\emptyset$ & $C_{2}$ \\
\hline$\Omega_{c}$ & $C_{2}$ & $C_{1}$ & $\Omega_{c}$ \\
\hline & $C_{2}$ & $\overline{C_{2}}$ & $\Omega_{c}$ \\
\hline
\end{tabular}

mass distribution in Figure 3 step d-3. The idea consists to divide the conflict into two components, a conflict due to the non-exhaustivity of the frame of discernment represented by the reject and an unknown conflict. In order to illustrate this idea, let us consider a two element frame of discernment $\Omega_{c}=\left\{C_{1}, C_{2}\right\} . \Omega_{c}$ can also be represented by $\left\{C_{1}, \overline{C_{1}}\right\}$ or $\left\{C_{2}, \overline{C_{2}}\right\}$. Given two BBA $m_{1}$ and $m_{2}$ which are defined on $\Omega_{c}$ by: $m_{1}=m_{1}\left(C_{1}\right), m_{1}\left(\overline{C_{1}}\right), m_{1}\left(\Omega_{c}\right)$ : a $3 \mathrm{D}$-object belongs or does not belong to the category $C_{1}$ or we are in a situation of almost complete ignorance concerning the category of that part. $m_{2}=m_{2}\left(C_{2}\right), m_{2}\left(\overline{C_{2}}\right), m_{2}\left(\Omega_{c}\right)$ : a $3 \mathrm{D}$-object belongs or does not belong to the category $C_{2}$ or we are in a situation of almost complete ignorance concerning the category of that part.

Here, $m_{1}$ and $m_{2}$ are considered as two independent sources of information to be combined in order to decide with which category, the object is associated. The evidence combination of these two belief using Dempsters rule of combination can be represented by Table I.

The last row and the first column of this table are named by the subsets of $\Omega_{c}$. Each of the squares in the table correspond to the intersection of the subset of each source of information $m_{1}$ and $m_{2}$. The value of BBA taken for the resulting subset is obtained by the multiplication of the BBA values of the subsets constituted $\Omega_{c}$. From this table, we can see that the conflict (the mass of the empty set) is represented by two grids. Its value is given by:

$$
m_{12}(\emptyset)=m_{1}\left(C_{1}\right) \cdot m_{2}\left(C_{2}\right)+m_{1}\left(\overline{C_{1}}\right) \cdot m_{2}\left(\overline{C_{2}}\right)
$$

The first portion of the conflict $m_{1}\left(C_{1}\right) \cdot m_{2}\left(C_{2}\right)$ is created because the two sources of information $m_{1}$ and $m_{2}$ related respectively to category $C_{1}$ and $C_{2}$ confirmed that a $3 \mathrm{D}$-object corresponds to the two categories at the same time. In contrast, the second portion of the conflict $m_{1}\left(\overline{C_{1}}\right) \cdot m_{2}\left(\overline{C_{2}}\right)$ is created because the two sources of information confirm that the 3D-object does not correspond to any category and thus the frame of discernment is not exhaustive. Hence, in our view, the separation between the first and the second portion of the conflict must be done because they do not have the same origin. We define a reject when sources of information confirm that the 3D-object does not correspond to any category. The reject is added to the frame of discernment as a new element and its belief degree is given by: $m_{12}($ reject $)=m_{1}\left(\overline{C_{1}}\right) \cdot m_{2}\left(\overline{C_{2}}\right)$. More specifically, in our case and through multiple sources of informations in the Table in Figure 3 step (d-2), the mass value of the reject is given by:

$$
m_{i}(\text { Reject })=\prod_{j=1}^{J}\left(m_{i j}\left(\overline{C_{j}}\right)\right)
$$

Finally, according to the pignistic probability deduced from the mass function computed in section 3.2, a decision can be taken about the reject of the 3D-object.

\section{EXPERIMENTAL RESULTS}

We present results from three experiments. In the first we evaluate the performance of the belief based classifier and explore the impact of the choice of descriptors on classifier accuracy. The accuracy is computed as the percentage of object models which 


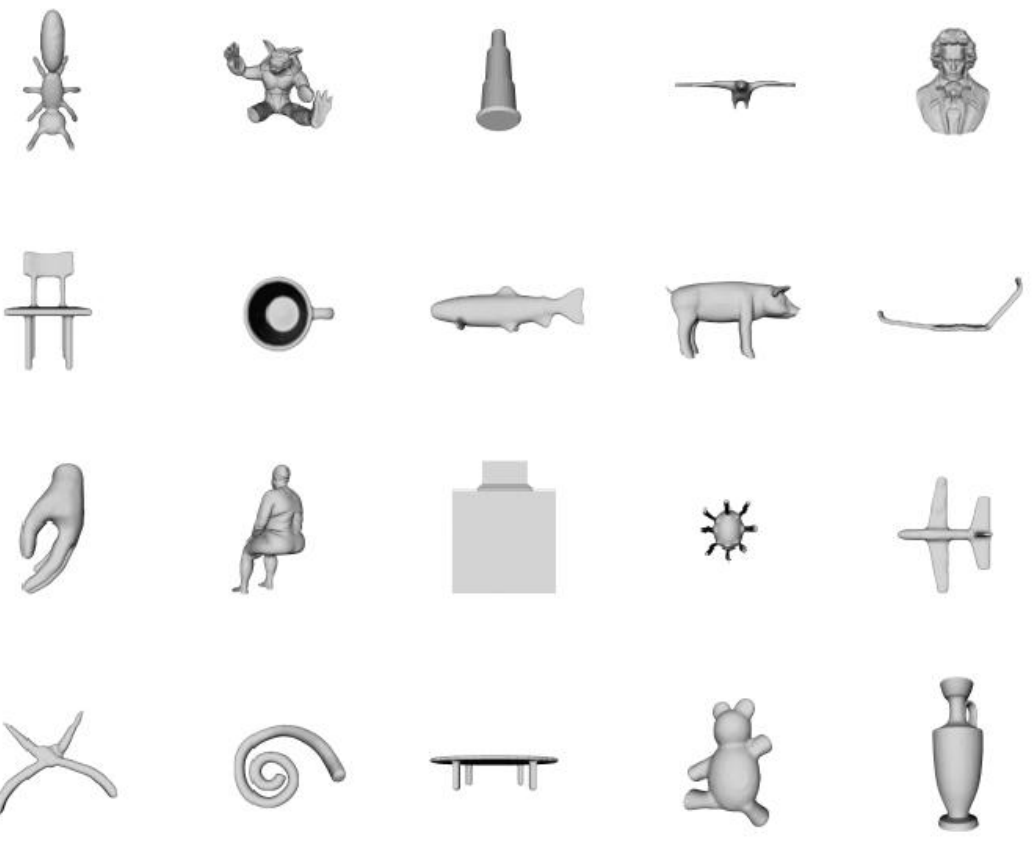

Fig. 4. Shrec07 dataset snapshot. Each object corresponds to one category.

are correctly classified. We then compare the performance of the belief based classifier with the Bayesian classifier [Huber et al. 2004] on the same problem. In the penultimate experiment we describe results with comparison to some state of the art retrieval methods. The last experiment shows the contribution of reject modeling option of our framework.

The experiments were conducted on two different datasets. The first dataset is the Shrec07 database. It contains $4003 \mathrm{D}$-objects classified into 20 classes. It is a challenging dataset, not only because of the large number of classes, but also because it contains shapes with highly variable poses and non-rigid or isometric transformations. Figure 4 shows some examples from this dataset. Each object in the figure represents one class. The second dataset is composed of shapes from the Tosca and the Sumner datasets. The Tosca dataset has been proposed by Bronstein et al. [Bronstein et al. 2007] for non-rigid shape correspondence measures. The Sumner dataset has been proposed by Sumner and Popovic [Sumner and Popovic 2004] for deformable shape correspondence. The total set size is 380 shapes. Figure 5 shows some examples from this dataset. Each object in the figure represents one class.

\subsection{Framework performance}

From a qualitative point of view, Figure 6 and 7 give a good overview of the efficiency of the framework on the Shrec07 dataset. Figure 6 presents a confusion matrix. Rows in this matrix correspond to query parts extracted from a human 3D-object, and columns correspond to the different categories shown in Figure 4 (Ordered from left to right and from top to bottom). The lightness of each element $(i ; j)$ is proportional to the magnitude of the similarity between the part $i$ and its closest representative one in the category $j$. Lighter elements represent better matches, while hot elements indicate 

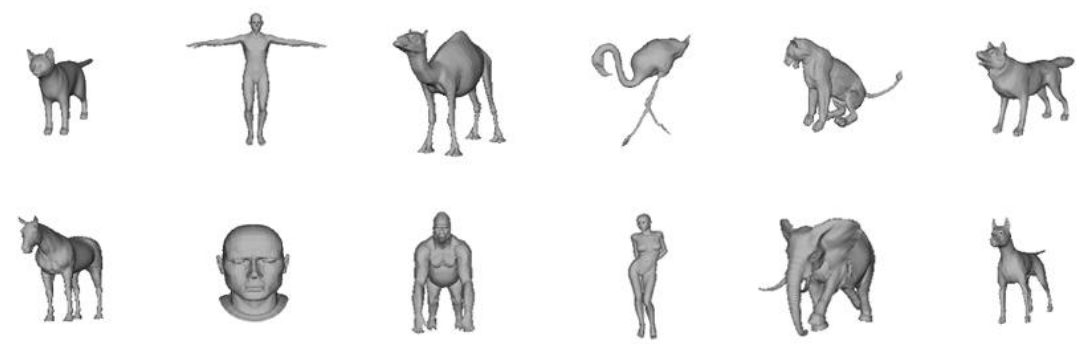

Fig. 5. Tosca-Sumner dataset snapshot. Each object corresponds to one category.

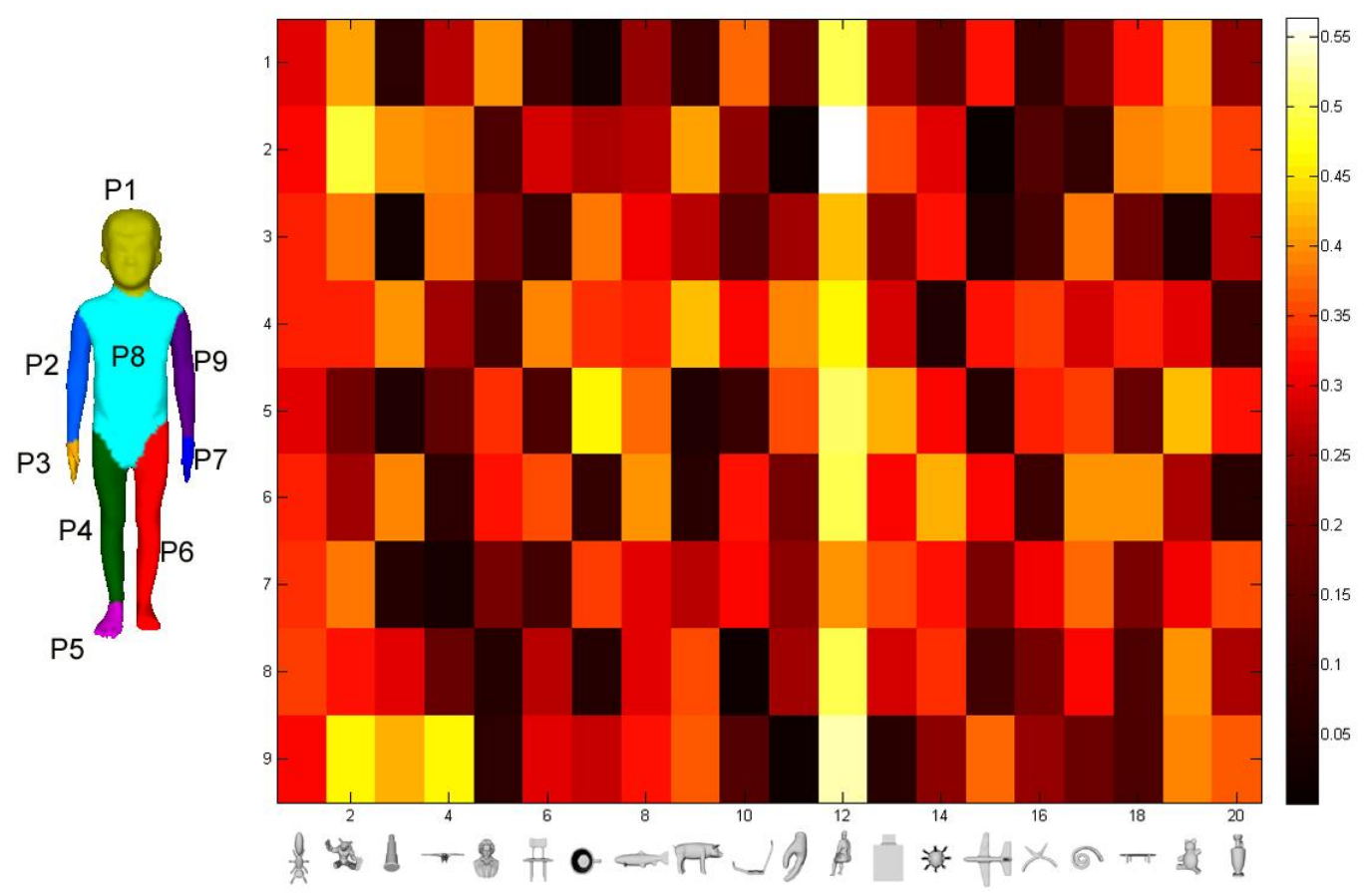

Fig. 6. The confusion matrix for a human object part categorization. Rows are query parts. Columns are object categories. (Using the GD2 feature descriptor on the Shrec07 dataset)

worse matches. One can notice in this visualization that the parts of the human object tends to match with the 12 th object category which corresponds to the human one in Figure 4. This result confirms our assumption that 3D-objects in the same category have the same parts.

Figure 7 shows another confusion matrix. In this matrix rows correspond to 3Dobject queries and columns correspond to the categories shown in Figure 4. The lightness of the diagonal squares of the matrix proves the effectiveness of our classifier.

More quantitatively, Table II and Table III show the classification results of our framework using different descriptors. On the Shrec07 dataset (Table II), HSI and Gcords descriptors yielded an accuracy of around 73\%, while the GD2 leads to a much higher accuracy of around $93.5 \%$. These results show that the GD2 is more suited for 


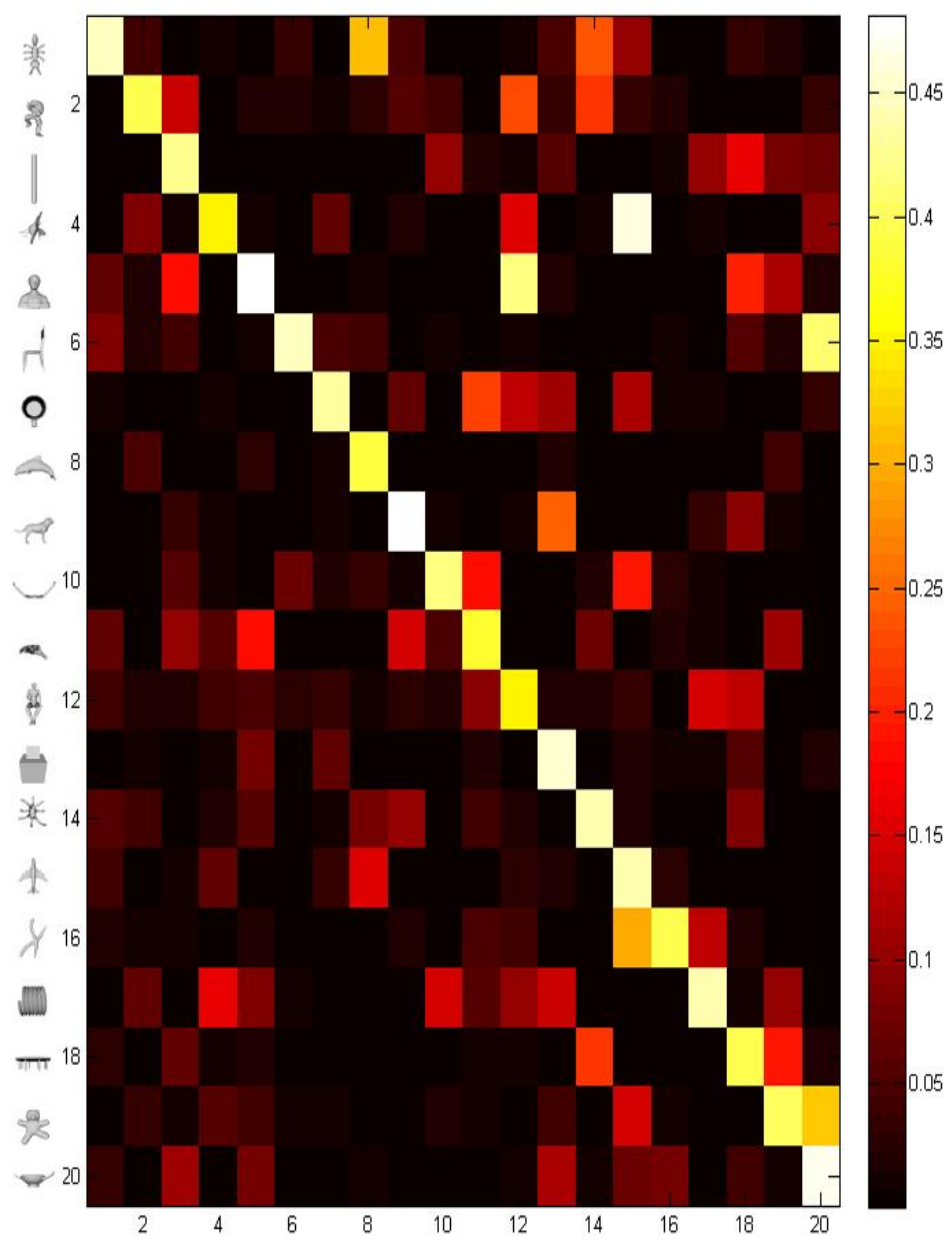

Fig. 7. The confusion matrix for some 3D-object categorization. Rows are query object. Columns are object categories. (Using the GD2 feature descriptor on the Shrec07 dataset)

Table II. Classification accuracies with feature descriptor changing on the Shrec07 dataset.

\begin{tabular}{|c|c|c|c|c|c|c|c|}
\hline Test Data & Dataset Size & GD2 & HSI & Gcords & GD2-HSI & GD2-Gcords & HSI-Gcords \\
\hline \hline Training Set & 100 & 96.7 & 75.6 & 74.3 & 96.7 & 96.7 & 90.9 \\
\hline Test Set & 300 & 90.3 & 70.8 & 69.9 & 92.9 & 91.1 & 78.5 \\
\hline Entire Dataset & 400 & 93.5 & 73.2 & 72.1 & 94.8 & 93.9 & 84.7 \\
\hline
\end{tabular}

Table III. Classification accuracies with feature descriptor changing on the Tosca-Sumner dataset.

\begin{tabular}{|c|c|c|c|c|c|c|c|}
\hline Test Data & Dataset Size & GD2 & HSI & Gcords & GD2-HSI & GD2-Gcords & HSI-Gcords \\
\hline \hline Training Set & 90 & 98.5 & 82.9 & 85.4 & 98.9 & 96.7 & 94.3 \\
\hline Test Set & 290 & 95.2 & 80.5 & 82.6 & 96.9 & 90.8 & 90.7 \\
\hline Entire Dataset & 380 & 96.85 & 81.7 & 84 & 97.9 & 93.75 & 92.5 \\
\hline
\end{tabular}


Table IV. Classification result comparison with the Bayesian classifier on the Shrec07. (Using the GD2-HSI feature descriptor).

\begin{tabular}{|c|c|c|c|}
\hline Test Data & Dataset Size & $\begin{array}{c}\text { Our methods } \\
\text { Accuracy (\%) }\end{array}$ & $\begin{array}{c}\text { Bayesian classifier } \\
\text { Accuracy (\%) }\end{array}$ \\
\hline \hline Training Set & 100 & 96.7 & 68.9 \\
\hline Test Set & 300 & 92.9 & 65.5 \\
\hline Entire Dataset & 400 & 94.8 & 66.63 \\
\hline
\end{tabular}

Table V. Classification result comparison with the Bayesian classifier on the Tosca-Sumner. (Using the GD2-HSI feature descriptor)

\begin{tabular}{|c|c|c|c|}
\hline Test Data & Dataset Size & $\begin{array}{c}\text { Our methods } \\
\text { Accuracy (\%) }\end{array}$ & $\begin{array}{c}\text { Bayesian classifier } \\
\text { Accuracy (\%) }\end{array}$ \\
\hline \hline Training Set & 90 & 98.9 & 77.9 \\
\hline Test Set & 290 & 96.9 & 73.4 \\
\hline Entire Dataset & 380 & 97.9 & 75,65 \\
\hline
\end{tabular}

local shape description. A combination of the GD2 and HSI feature yields a better accuracy than GD2 feature alone $94.8 \%$ (the combination is based on the mean distance). On the Tosca-Sumner dataset (Table III), the combination of the GD2 and HSI feature also gives the highest accuracy rate $97.9 \%$.

\subsection{Belief classifier versus Bayesian classifier}

Table IV and Table V show a comparison accuracy between the belief classifier and the Bayesian classifier on respectively the shrec07 and the Tosca-Sumner datasets. On the shrec07 dataset, our classifier showed an accuracy of $96.7 \%$ and $92.9 \%$ on the training set and an independent test set, respectively. That is to say $94.8 \%$ accaracy over the entire dataset. Using a Bayesian classifier we report only $66.63 \%$ accurancy. On the Tosca-Sumner dataset, results comfirme the contribution of the use of the belief framework instead of the Bayesian one. The belief classifier reports $97.9 \%$ while the Bayesian one reports only $75.65 \%$.

\subsection{Comparison with related work}

In this experiment we compare our method effectiveness to methods proposed by Biasotti et al. [Biasotti et al. 2006]. In their work, authors compared the performance of five similarity measures on four different shape descriptors in classifying 3D objects. The four different shape descriptors used in their paper are: The spherical harmonics (SH) in [Kazhdan et al. 2003] which is a volume-based descriptor, The light-field descriptor (LF) in [Chen et al. 2003] which is an image-based descriptor and two topological matching methods, the Multi-resolution Reeb graph in (MRG) [Hilaga et al. 2001] and the Extended Reeb graph (ERG) in [Biasotti and Marini 2005].The five similarity measures are: The Minimum Distance Classifier (MinDC) which coincides with the Nearest Neighbor classifier, Maximum Distance Classifier (MaxDC) which classifies a query by taking into account the most dissimilar descriptor belonging to the class. The Average Distance Classifier (AvgDC) which is defined as the average distances between the query and the members of the class. The Centroid Distance Classifier (CDC) where the query is classified according to its dissimilarity with a representative member of a class. And the Atypicity Distance Classifier (ADC) which evokes the notion of typicity, to represent how much a descriptor is typical of the class it belongs to, with respect to the elements in the other classes.

In order to demonstrate the effectiveness of our method compared with the Biasotti et al's classifiers, we tested our method on the same dataset used by Biasotti et al. This dataset is a subset of the SHREC07 dataset composed of 280 3D-objects classified into 14 classes. The results of the experiment are shown in Table VI. Each entry is related 
Table VI. Classification result comparison with related work.

\begin{tabular}{|c|c|c|c|c|}
\hline \multirow{2}{*}{} & \multicolumn{4}{|c|}{ State of the art methods (Rate\%) } \\
\cline { 2 - 5 } & SH & LF & MRG & ERG \\
\hline \hline MinDC & $89 \%$ & $88 \%$ & $88 \%$ & $83 \%$ \\
\hline MaxDC & $33 \%$ & $38 \%$ & $41 \%$ & $38 \%$ \\
\hline AvgDC & $66 \%$ & $73 \%$ & $74 \%$ & $58 \%$ \\
\hline CDC & $64 \%$ & $68 \%$ & $76 \%$ & $60 \%$ \\
\hline ADC & $63 \%$ & $68 \%$ & $73 \%$ & $58 \%$ \\
\hline \multicolumn{5}{|c|}{ Our method (Rate\%) } \\
\hline \multicolumn{5}{|c|}{$97.6 \%$} \\
\hline
\end{tabular}

Table VII. Classification results with Reject option on the Shrec07 dataset.

\begin{tabular}{|c|c|c|c|c|}
\hline $\begin{array}{c}\text { Classification } \\
\text { Algorithm }\end{array}$ & $\begin{array}{c}\text { Training } \\
\text { Set Size }\end{array}$ & $\begin{array}{c}\text { Test } \\
\text { Set Size }\end{array}$ & $\begin{array}{c}\text { Reject } \\
\text { Rate (\%) }\end{array}$ & $\begin{array}{c}\text { Accuracy } \\
(\%)\end{array}$ \\
\hline \hline Our algorithm with reject option & 100 & 400 & 6.6 & 96.5 \\
\hline Bayesian algorithm with reject (T=0.1) & 100 & 400 & 3.1 & 68 \\
\hline Bayesian algorithm with reject (T=0.15) & 100 & 400 & 7.3 & 70.7 \\
\hline Our algorithm without reject option & 100 & 400 & 0 & 94.8 \\
\hline Bayesian algorithm without reject & 100 & 400 & 0 & 66.63 \\
\hline
\end{tabular}

to the performance of a given shape descriptor (enumerated in the second row) for a given classifier (reported in the first column of the table). The classification accuracy of our method is given in the last row. While Biasotti et al. concluded that the MinDC (nearest neighbor) similarity measure performed the best for all four different shape descriptors in their work, one can notice that our classifier shows the highest classification rate $97.6 \%$. Moreover, the nearest neighbor based approaches require to compare each object to be classified to all objects in the dataset, which seems to be impractical with huge databases where our method is preferred, while it requires matching of only the representative parts. Please note that using a PC with a $3 \mathrm{Ghz}$ Core 2 Duo processor with 3 GB memory, and a Matlab implementation of our algorithms, the running time of the labeling process depends on the quality of the meshes and their number of vertices. The full processing time of a query (from the SHREC07 or the Tosca-Sumner datasets) varies from 2 to 25 seconds.

\subsection{Reject option contribution}

Table VII shows the accuracies for 3D-classification with and without reject option on the Shrec07 dataset. One can notice that the classification accuracy improved from $94.8 \%$ to $96.5 \%$. For Bayesian classifier, the 3D-object whose maximum a posteriori probability is below the threshold ( $\mathrm{T}$ ) in Table VII are rejected. When $\mathrm{T}=0.15$, we notice that the Bayesian classifier rejects much more than our classifier. However our accuracy is still higher.

\section{CONCLUSION}

In this paper, we have presented a parts-based method for categorizing 3D-objects using a new evidential classifier. The categorization process is completely automated and consists of two different stages. The training stage which lies on the category model building is based on the belief function theory and it goes into two steps: 1) 3D-object partitioning and 2) representative parts construction. The second stage is the labeling, in which belief functions have been also used. In the labeling process, we have introduced a reject option, which can be used to handle the labeling of unknown 3D-objects. The classifier has been evaluated on two databases of 400 and 380 3D-models. Our system achieved a classification accuracy over $94.8 \%$ and $97.9 \%$, respectively on the two datasets. The reject option has also been evaluated and the experimental results 
obtained on the Shrec07 dataset show that this option efficiently improves the classification accuracy from $94.8 \%$ to $96.5 \%$. However, the surface partitioning method used in our framework introduces a bias in the categorizing process. To guarantee stability and performance, this partitioning has to be stable within a same class of objects. In practice, with the SHREC07 and Tosca-Sumner datasets, partitioning turns out to be homogeneous within most classes. Furthermore, since we have focused our interest to propose meta-algorithm for a relevant categorizing approach, some parts of the method can be changed and tested (3D partitioning, local descriptors). For example, in order to deal with imperfect meshes, we can use volume-based partitioning approaches [Mademlis et al. 2008] which are preferred than surface-based approaches. In the future, we would like to investigate the integration of the spatial relation between parts in the matching process.

\section{ACKNOWLEDGMENTS}

This research was supported partially by the Contrat de Projet Etat-Région (CPER) Région Nord-Pas-De calais Ambient Intelligence

\section{REFERENCES}

Antini, G., Berretti, S., Del Bimbo, A., And Pala, P. 2005. Retrieval of 3d objects using curvature correlograms. In IEEE International Conference on Multimedia \& Expo.

Assfalg, J., Bertini, M., Del Bimbo, A., AND Pala, P. 2007. Content-based retrieval of 3-d objects using spin image signatures. IEEE Transactions on Multimedia 9, 589 - 599.

Biasotti, S., Giorgi, D., MArini, S., Spagnuolo, M., AND FAlCidieno, B. 2006. A comparison framework for $3 \mathrm{~d}$ object classification methods. In Multimedia content representation, classification and security. Vol. 4105. 314-321.

BiAsotTi, S. AND MARINI, S. 2005. 3d object comparison based on shape descriptors. International Journal of Computer Applications in Technology 23, 57-69.

Bronstein, A., Bronstein, M., Bruckstein, A., And Kimmel, R. 2008. Partial similarity of objects, or how to compare a centaur to a horse. International Journal of Computer Vision.

Bronstein, A., Bronstein, M., CARmon, Y., AND Kimmel, R. 2009. Partial similarity of shapes using a statistical significance measure. IPSJ Trans. Computer Vision and Application 1, 105-114.

Bronstein, A., Bronstein, M., AND Kimmel, R. 2007. Efficient computation of isometry-invariant distances between surfaces. IEEE Trans. Visualization and Computer Graphics 13/5, 902-913.

Chen, D.-Y., Tian, X.-P., Shen, Y.-T., And Ouhyoung, M. 2003. On visual similarity based 3D model retrieval. Eurographics 22, 223-232.

Donamukkala, R., Huber, D., Kapuria, A., And Hebert, M. 2005. Automatic class selection and prototyping for 3-d object classification. 3-D Digital Imaging and Modeling (3DIM).

Filali Ansary, T., DaOudi, M., AND VAndeborRe, J.-P. 2007. A bayesian 3D search engine using adaptive views clustering. IEEE Transactions on Multimedia 9, 1 (January), 78-88.

Funkhouser, T., Min, P., Kazhdan, M., Chen, J., Halderman, A., And Dobkin, D. 2003. A search engine for 3D models. ACM Transactions on Graphics 22, 1 (January), 83-105.

Hilaga, M., Shinagawa, Y., Kohmura, T., AND Kunit, T. 2001. Topology matching for fully automatic similarity estimation of 3D shapes. In SIGGRAPH. 203-212.

Huber, D., Kapuria, A., Donamukkala, R., And Hebert, M. 2004. Parts-based 3d object classification. In IEEE Conference on Computer Vision and Pattern Recognition (CVPR).

Kazhdan, M., Funkhouser, T., And RusinkiewiCZ, S. 2003. Rotation invariant spherical harmonic representation of $3 \mathrm{~d}$ shape descriptors. Geometry Processing, Aachen, Germany.

KoEnderink, J. AND VAN Doorn, A. 1992. Surface shape and curvature scales. Image and Vision Computing 10, 557-565.

Mademlis, A., Daras, P., Axenopoulos, A., Tzovaras, D., And Strintzis, M. 2008. Combining topological and geometrical features for global and partial 3-d shape retrieval. Multimedia, IEEE Transactions on 10, 5 (aug), $819-831$.

Osada, R., Funkhouser, T., Chazelle, B., And Dobkin, D. 2002. Shape distributions. ACM Transactions on Graphics 21(4), 807-832. 
Paquet, E. AND Rioux, M. 1999. Nefertiti: a query by content system for three-dimensional model and image databases management. Image and Vision Computing 17, 157-166.

Shafer, G. 1976. A mathematical theory of evidence. Princeton University Press.

Shamir, A. 2008. A survey on mesh segmentation techniques. Computer Graphics Forum 27, 6, 1539-1556.

Sumner, R. AND Popovic, J. 2004. Deformation transfer for triangle meshes. In International Conference on Computer Graphics and Interactive Techniques.

Tierny, J., VANDEBorRe, J.-P., AND DAOUdi, M. 2007. Topology driven 3D mesh hierarchical segmentation. In IEEE International Conference on Shape Modeling and Applications (SMI 2007). Lyon, France, $215-220$.

VAilaya, A., Figueiredo, M.-A.-T., JAin, A.-K., AND ZHANG, H.-J. 2001. Image classification for contentbased indexing. IEEE Transactions on Image Processing 10, 117-130.

ZouhAL, L.-M. AND DENOEUX, T. May 1998. An evidence-theoretic k-nn rule with parameter optimization. IEEE Transactions on Systems 28, 263-271. 